\title{
Research
}

\section{Nasal balloon autoinflation for glue ear in primary care:}

\author{
a qualitative interview study
}

\begin{abstract}
Background

Nasal balloon autoinflation is an effective, nonsurgical treatment for symptomatic children with glue ear, although uptake is variable and evidence about acceptability and feasibility is limited.
\end{abstract}

\section{Aim}

To explore parent and healthcare professional views and experiences of nasal balloon autoinflation for children with glue ear in primary care.

\section{Design and setting}

Qualitative study using semi-structured interviews with a maximum-variety sample of parents, GPs, and practice nurses. The study took place between February 2013 and September 2014.

\section{Method}

Semi-structured face-to-face and telephone interviews were audiorecorded, transcribed verbatim, and analysed using inductive thematic analysis.

\section{Results}

In all, 14 parents, 31 GPs, and 19 nurses were included in the study. Parents described the nasa balloon as a natural, holistic treatment that was both acceptable and appealing to children. GPs and nurses perceived the method to be a lowcost, low-risk strategy, applicable to the primary care setting. Good instruction and demonstration ensured children mastered the technique and engaged with the treatment, but uncertainties were raised about training provision and potential impact on the GP consultation. Making nasal balloon autoinflation part of a child's daily routine enhances compliance, but difficulties can arise if children are unwell or refuse to cooperate.

\section{Conclusion}

Nasal balloon autoinflation is an acceptable, low-cost treatment option for children with glue ear in primary care. Provision of educational materials and demonstration of the method are likely to promote uptake and compliance. Wider use of the nasal balloon has the potential to enhance early management, and may help to fill the management gap arising from forthcoming changes to care pathways.

\section{Keywords}

general practitioners; glue ear; otitis media with effusion; parents; primary care; qualitative research.

\section{INTRODUCTION}

Otitis media with effusion (OME), or glue ear, is the commonest chronic condition of childhood, affecting $80 \%$ of children by the age of 8 years. ${ }^{1}$ The vast majority of such children are seen in primary care. Because traditional or historic medical treatments, including antibiotics, ${ }^{2-4}$ have been largely ineffective, it has led to the prevailing view that $\mathrm{OME}$ is really a surgical condition. ${ }^{5}$ A forthcoming report from NHS England rationalising ineffective and risky treatments has identified grommet surgery as only suitable for children who strictly meet the National Institute for Health and Care Excellence (NICE) criteria, or in exceptional cases. ${ }^{6}$ Although a move away from surgery is to be welcomed, glue ear clearly has significant impacts on children's communication skills and quality of life, 7,8 and the problem remains about what to do for those who are clinically affected. The only proven effective non-surgical intervention suitable for primary care is nasal balloon autoinflation; $;, 10$ the authors believe that this has the potential to positively contribute to the early management of children with $\mathrm{OME}$ in the community setting.

Despite being available on prescription and over-the-counter for $>10$ years, uptake and usage of the nasal balloon is still relatively low, with around 17000 annual prescriptions in the UK, ${ }^{11}$ compared with around 33000 surgical procedures for grommets. ${ }^{12}$ Little is yet known about the potential barriers and facilitators to such treatment, and no previous qualitative work has been carried out to explore the real-life practicalities of prescribing and implementing the nasal balloon method during the NICE recommended 3-month monitoring period.

This study sets out to address how nasal balloon autoinflation can be taken up more widely in the NHS by learning from the experiences of an important sample of healthcare providers and parents of children who took part in the autoinflation randomised study (AIRS), ${ }^{10}$ and who are considered early adopters of the method. AIRS was a large UK primary care trial set in $43 \mathrm{GP}$ practices in the UK, in which 320 school children aged 4-11 years with OME were randomised to either nasal balloon autoinflation plus usual care, or usual care alone, for 1-3 months. The study found that regular use of the nasal balloon was effective in both clearing middle-ear effusions $149.6 \%$ versus $38.3 \%$ with normal tympanogram at 3 months, number needed to treat $=9$ ] and improving symptoms and ear-related quality of life. GPs were the principal investigators and provided local oversight and study support to the nurses. Nurses were the main study recruiters and received face-to-face training in study methods and tympanometry, and brief instruction in the nasal balloon method. Children randomised to the nasal balloon arm received brief instructions and/or a demonstration from

Email: j.vennikdsoton.ac.uk

Submitted: 8 August 2018; Editor's response: 21 August 2018; final acceptance: 4 September 2018.

\section{(CBritish Journal of General Practice}

This is the full-length article (published online 4 Dec 2018) of an abridged version published in print. Cite this version as: $\mathbf{B r} \mathbf{J}$ Gen Pract 2018; DOI: https://doi.org/10.3399/bjgp18X700217 


\section{How this fits in}

Glue ear is the commonest condition of childhood, significantly affecting communication skills and quality of life. Limited medical options are available and a forthcoming review of care pathways, including grommet surgery, contributes to the dilemma of how to best manage affected children in primary care. Nasal balloon autoinflation is an effective but still relatively unknown treatment option. This study found the autoinflation method to be acceptable and feasible in primary care, and highlighted important strategies for implementation. The authors suggest that wider use has the potential to improve early management of affected children seen in general practice.

the nurse, and families were provided with a link to a trial demonstration video. In the current qualitative work, the authors also explore the views and perceptions of a sample of GPs not previously exposed to the nasal balloon method who are therefore likely to represent wider NHS practice.

\section{METHOD}

\section{Participants and procedures}

Parents of 70 children who had completed the AIRS study in the preceding 6 months were approached by mail via their GP practice and asked to express interest by returning a reply slip to the study team. The study took place between February 2013 and September 2014. Interested parents were purposefully sampled for a maximum range of their child's characteristics, including age, sex, baseline severity of OME as determined by tympanometry, GP practice location, and AIRS treatment allocation (nasal balloon autoinflation plus usual care, or usual care alone).

A maximum-variety sample of GPs was identified, and included AIRS investigators with previous experience of the nasal balloon and GPs with no prior exposure to the treatment method, thus representing wider NHS practice. GPs who were AIRS investigators were invited to participate via email directly from the study team. The National Institute for Health Research (NIHR) Clinical Research Network invited expressions of interest from GPs via their newsletter and through personal contact. GP practices from regions of higher social deprivation not represented in the initial sample were invited by mail by the study team. Responders were purposefully sampled to include a maximum variation of practice sociodemograhics, practice location, and previous experience using the nasal balloon.

Practice nurses who were recruiters for AIRS were approached by the study team, and a convenience sample of responders was included in the study.

Full details of the sampling and recruitment process are presented in Figure 1.

\section{Interviews}

Written consent was given by all participants before interview. Semi-structured interviews were conducted either face-to-face or by telephone by a trained interviewer, each lasting approximately 30 minutes. The interviewer was trained in qualitative research methods and was also the AIRS trial manager, so had previously worked with the AIRS GPs and research nurses but was unknown to the parent participants and the non-recruiting GPs. Interview guides for patients and healthcare professionals, developed through literature review and collaboration with the AIRS research team, were used to guide the interviews (further information is available from the authors on request). This facilitated the exploration of key issues, allowing the process to remain sufficiently flexible to explore unexpected topics or concerns. GPs unfamiliar with the nasal balloon method were provided with a link to the AIRS instruction video before the interview.

The interviews were digitally audiorecorded and transcribed verbatim. Any identifiable data were removed to preserve participant anonymity.

\section{Analysis}

Data were managed using NVivo (version 10) software, and the transcripts from each participant group (parents, GPs, and nurses) were independently analysed using inductive thematic analysis. ${ }^{13}$ The analysis commenced with familiarisation with the transcripts from each participant group, and they were then systematically and comprehensively coded using open coding. This is a method of reducing the data while capturing the semantics and concepts within the data itself. The first three transcripts in each group were coded by two coders, and a coding framework agreed. Codes were refined into broad themes, both inductively and guided by a priori knowledge of the topic area. The themes and sub-themes of each analysis (parents, nurses, and GPs) were compared and contrasted, and overarching themes were developed. Themes were then defined 

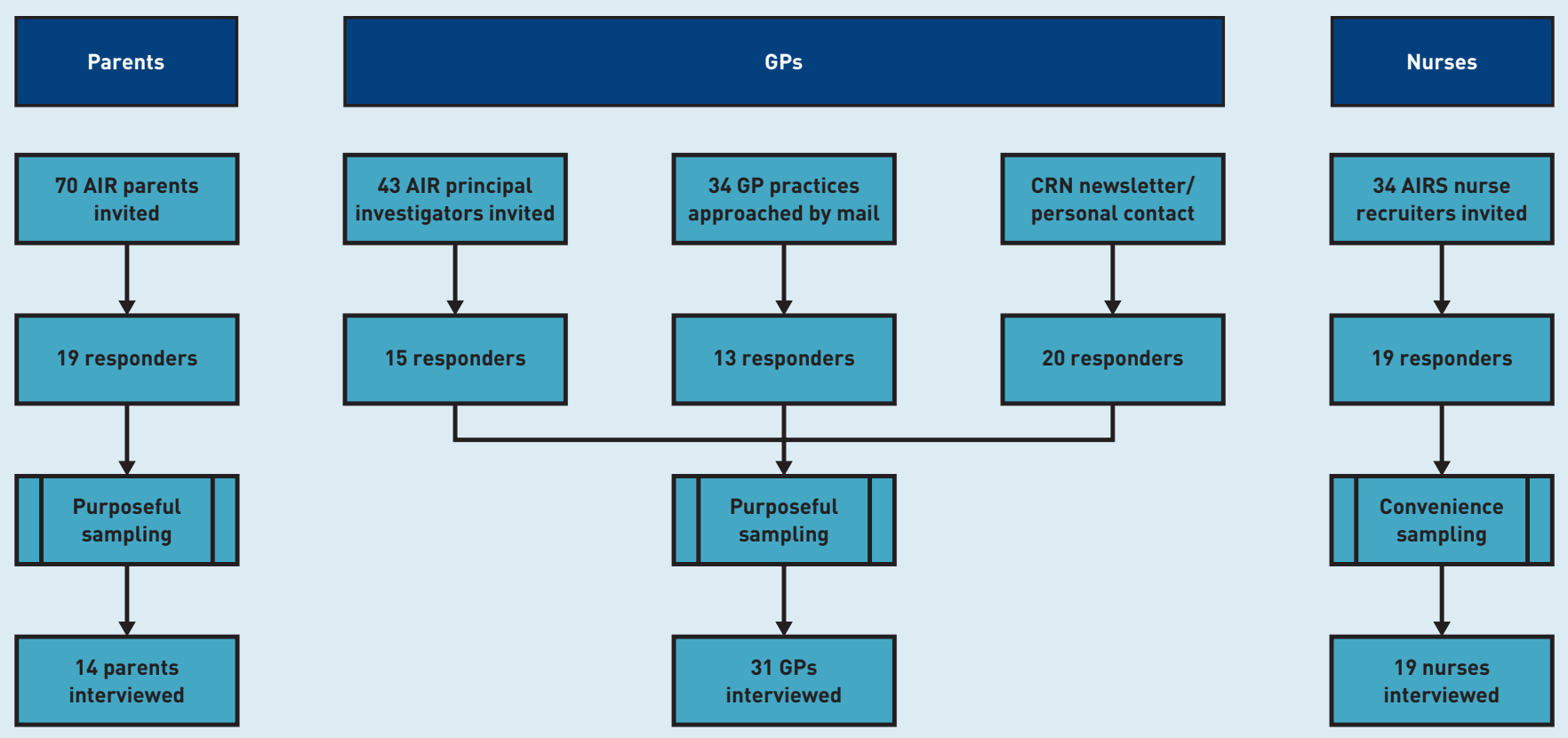

Figure 1. Identifying, sampling, and recruiting participants. AIRS = autoinflation randomised study. CRN = Clinical Research Network and described in relation to the research questions and the existing literature.

\section{RESULTS}

\section{Participants}

A total of 14 parents, $31 \mathrm{GPs}$, and 19 nurses from 18 GP practices in Wessex, West of England, Thames Valley, and Cheshire participated in a research interview. Characteristics are presented in Table 1.

\section{Themes}

Three key themes emerged from the analysis (Box 1). These themes are not an exhaustive account of the findings, but substantially represent the major themes interpreted to be relevant in exploring wider feasibility and acceptability of nasal balloon autoinflation in primary care.

\section{Theme 1: Perceptions of the nasal balloon}

Coherence and acceptability. Nasal balloon autoinflation was described by most parents as a natural, holistic treatment that offers a practical solution to glue ear and is appealing to children:

Anything holistic that doesn't involve sort of medicine or drugs, I think, is brilliant. So that's what attracted me to the study, because it's very practical, and a physical, practical solution.' (Parent 3)

GPs with previous experience of the nasal balloon method described it as easy to understand and explain to families, having a logical mode of action similar to methods of middle-ear inflation that were currently being recommended in some GP practices; for example, the Valsalva manoeuvre, mouth inflation of party balloons:

I think that would be something really easy and something that you could show to parents actually in the appointment.'(GP 19)

GPs and practice nurses described nasal balloon autoinflation as a low-harm, lowcost intervention appropriate for primary school age children, which could promote self-management and enhance the watchful waiting process:

I think while they are waiting for the audiological review is a good starting point, because if it's already improving by then, it reinforces the concept of watchful waiting. (GP 13)

'I think with the balloon, I would assume it's relatively inexpensive. It has to be a good process of elimination for glue ear and grommets, and I think a lot of referrals to consultants at, you know, secondary care, could be avoided by 3 months of trialling the balloon.' (Nurse 1)

Credibility. Some GPs, particularly those who were unfamiliar with the nasal balloon before this study, raised concerns about the credibility of prescribing or recommending such a treatment, describing the nasal balloon as possibly a 'gimmick', or purely a placebo": 


\section{Table 1. Participant characteristics}

\begin{tabular}{|c|c|}
\hline Parent participants & $n=14$ \\
\hline Female & 14 \\
\hline \multicolumn{2}{|l|}{ Education level (highest qualification achieved) } \\
\hline - School to 16, GCSEs/O level & 4 \\
\hline - A level & 2 \\
\hline - Highers, Scotvec, or NVQ & 1 \\
\hline - University/professional/postgraduate degree & 7 \\
\hline \multicolumn{2}{|l|}{ Child characteristics ratio $(n=14)$} \\
\hline - Male/female & $7 / 7$ \\
\hline - Age group $(<6.5$ years $/>6.5$ years $)$ & $13 / 1$ \\
\hline - Baseline severity of OME (one ear/two ears) & $10 / 4$ \\
\hline - AIRS treatment allocation (nasal balloon/usual care) & $10 / 2$ \\
\hline GP participants & $n=31$ \\
\hline Ratio male/female & $24 / 7$ \\
\hline Mean years in general practice (range) & $15.10(7-22)$ \\
\hline Mean practice IMD: 1 low, 10 high (range) & $8.1(3-10)$ \\
\hline Mean list size (range) & 10951 (3164-28 827) \\
\hline \multicolumn{2}{|l|}{ Practice location } \\
\hline - Rural town and fringe & 3 \\
\hline - Rural village and dispersed & 3 \\
\hline - Urban city and town & 20 \\
\hline - Urban major conurbation & 5 \\
\hline Ratio AIRS recruiters/non-recruiters & $12 / 19$ \\
\hline Nurse participants & $n=19$ \\
\hline Female & 19 \\
\hline Mean years in current role (range) & $9.1(3-20)$ \\
\hline Mean practice list size (range) & $10877(3378-28261)$ \\
\hline Mean practice IMD: 1 low, 10 high (range) & $8.0(6-10)$ \\
\hline
\end{tabular}

\section{Box 1. Themes and sub-themes}

$\begin{array}{ll}\text { Themes } & \text { Sub-themes } \\ \begin{array}{l}\text { 1. Perceptions of } \\ \text { the nasal balloon }\end{array} & \text { - Coherence and } \\ & \text { acceptability } \\ & \text { - Credibility } \\ \text { 2. Safety } & \text { - Suitability and } \\ \text { in primary care } & \text { relevance } \\ & \text { - Engaging families } \\ & \text { - Training and } \\ & \text { demonstration } \\ \text { - Mastering the } & \text { technique } \\ \text { 3. Continuing and } & \text { - Remembering and } \\ \text { monitoring } & \text { persevering } \\ & \text { - Monitoring }\end{array}$

When you look at it, it's just a load of balloons and a plastic device, and they will probably think, well, "what's the doctor telling me?" It might look a bit strange. (GP 12)

However, parents who participated in AIRS did not identify problems with credibility, and generally described the treatment as a practical and holistic treatment for glue ear:

I was really keen to see whether this would work, because this is a very non-invasive way of dealing with glue ear, I thought.' (Parent 7)

Safety. Parents and healthcare professionals expressed some uncertainties about potential harms associated with inflating the nasal balloon. Parents noticed that some children turned red in the face, whereas others reported children experiencing some mild pain, popping, and clicking when using the balloon:

Sometimes, I did kind of wonder, thinking, you know, it puts a lot, it's what the pressure of it will be doing in your head, you know. His face would go quite red.' (Parent 9)

However, most GPs described the balloon as a low-harm treatment option, and nurses did not report any particular safety concerns in this study:

'It's easy to use. Physiologically, you can see how it could be helpful ... and the likelihood of it doing any harm is very, very low. 'GP 1)

\section{Theme 2: Implementing in primary care}

Suitability and relevance. A common concern raised by GPs, especially those who did not have personal experience with the balloon, was how to select children for nasal balloon treatment. There was a general perception that the treatment would be suitable for older children (5-7 years), as the technique was thought to require a level of dexterity and cooperation:

You wonder what age onwards the child would be able to do that, but you would have thought from about 5 onwards, maybe; I would have thought a child under 5 might struggle. '(GP 16)

However, nurses and GPs with previous experience of the nasal balloon found that children aged $\geq 4$ years were generally capable of successfully inflating the balloon with some training.

Some GPs suggested potential barriers to uptake of the nasal balloon in diverse cultural populations and areas of increased social deprivation:

We are in an urban practice, with lots of different migrants registered at our practice, so language is one barrier; cultural expectation is another barrier.' (GP 28)

Engaging families. GPs described the perceived need for a high level of parental motivation to ensure good uptake with the nasal balloon:

'The other barrier would be patient acceptance; do they want to take it up? And they might go "oh, I can't be bothered with this sort of thing, it's too much of a faff". ' (GP 7)

Nurses described the need for children to be willing to cooperate, as the technique 
requires active participation:

'It's not like taking a tablet, is it? It's something that they've got to do, and I think that's always hard for a parent, isn't it, getting a child to do something, rather than just, you know, take something?'(Nurse 12)

Although some GPs proposed that a lack of parental commitment and child cooperation could be a barrier to engagement, the vast majority of parents in the study were happy to try the nasal balloon as part of AIRS, and readily committed to the treatment regimen. Additionally, children were engaged with the treatment, especially in the first few weeks, if it was made fun or part of a game:

'The girls thought that was great fun: anything to do with balloons, isn't it? They think it's great.' (Parent 5)

Training and demonstration. GPs proposed that a good demonstration would be required to ensure children mastered the nasal balloon technique and engage with the intervention:

'Demonstrations are always really important. It's all about technique. All these things are about technique.' (GP 30)

Nurses agreed that either demonstrating the technique personally, or asking parents to demonstrate, was an effective way of encouraging and engaging the child:

I demonstrated, and they would then have a go and they, obviously, weren't particularly good at it. So I said to the mum, "oh, you have a go and if you can do it, that helps the child". (Nurse 12)

Parents also described the importance of a good demonstration to help them understand exactly what their child needed to do:

It was very easy. The instructions were straightforward; the nurse gave us great demonstrations and a great explanation about how to use it.' (Parent 11)

However, although some GPs proposed that a personal demonstration would add credibility to the treatment and improve the technique, others either did not see demonstrating the nasal balloon as their role, or did not consider it feasible during a routine consultation:

I honestly think I would struggle to teach it within a busy consultation, because there are often other problems being brought, or other kids running round the room, and I don't think I would be the best person to teach it.' (GP 31)

Some GPs suggested that an online training video ought to be sufficient to train families in the use of the nasal balloon:

'I wouldn't necessarily feel the need to have to demonstrate it, because these days I think you should get a video or YouTube, or whatever, to show it, I'd have thought.' (GP 5)

Others suggested that pharmacists could demonstrate the balloon as part of their dispensing procedure, although there were uncertainties about whether they would be happy to fulfil this role:

I find this with inhalers, that I'm very keen to get the pharmacist involved. So, if the pharmacist could actually demonstrate it, they've got a bit more time.' (GP 15)

Mastering the technique. Parents reported that some children have initial difficulties with inflating the nasal balloon. Nurses agreed that first inflations could be difficult for children due to the balloon tension, and some children are reported as having difficulties blowing through their nose. Inflations were reported as becoming easier if the balloon is pre-stretched by hand or mouth beforehand:

'I think they all found it a little bit difficult at the start, because the balloon was, and it is quite hard to blow up initially.' (Nurse 4)

Nurses described the importance of involving the children early on in the consultation process and giving lots of encouragement to achieve successful inflation:

'Even if they blew it up a bit, then we sort of said: "Oh, that's brilliant." And then, of course, the next time you saw them they'd be blowing it up to the size of an orange.' (Nurse 2)

Parents and nurses both reported that in most cases children either mastered the technique quickly or got better with practice:

She struggled a little bit at the beginning. But we then took it home and practised. Well, once she got the hang of it everybody who came in the door had to have it demonstrated.'(Parent 2) 


\section{Theme 3: Continuing and monitoring}

Remembering and persevering. Treatment with the nasal balloon is ideally 3 months a reasonable watchful waiting period - and thus requires an element of remembering to use it and persevering with treatment. GPs stated that this might cause problems for some families:

'There is always some element of complex family dynamics, and not everybody's on board with certain treatments that have to be repeated day in, day out.' (GP 19)

Parents, however, reported that making the nasal balloon part of a child's daily routine helped with adherence to the treatment regimen:

In the morning, whatever we were doing, and then at bedtime. So, it was just like cleaning your teeth, just brought it in as an extra thing to do as part of the routine. (Parent 6)

Parents and nurses reported that the use of incentives, such as sticker reward charts, improved compliance, and motivated children to use the nasal balloon:

I think, in general, the children love stickers of any sort, don't they? And if they can put a "well done" at the end of the day, because they've done it and inflated it, and it does give them a boost.' (Nurse 7)

Although many children managed and persevered in the AIRS trial, some nurses reported that children could lose interest over time:

'The first 2 or 3 weeks were fairly good, but once the children started getting bored with it, then it was a little bit hit-and-miss. (Nurse 13)

Additionally, a number of parents reported that children were less likely to comply with treatment if they found the technique difficult or uncomfortable, or if they were unwell:

It got trickier as the trial went on, because she got fed up with it; and she did get quite poorly a couple of times, with really bad colds and blocked up nose, and she just point blank refused. '(Parent 5)

Monitoring. GPs described relying on parents to monitor their child's hearing and to return to the GP if things had not improved. However, some parents remained unclear about how long to continue treatment, how quickly they should expect to see results, and if or when to return to the GP for further advice:

'So, how long do you persist with this before you think: "OK, I need to take it to the next level?" That isn't clear to me.' (Parent 8)

GPs without previous exposure to the nasal balloon described needing to build up personal experience with it in their own patient population:

'I think, if it didn't seem to, you know, if the vast majority were coming back with it not making any difference, it would probably fall out of favour for me, to be honest.' (GP 19)

\section{DISCUSSION}

\section{Summary}

Parents described the nasal balloon as a natural, holistic treatment that was both acceptable and appealing to children. GPs and nurses perceived the method to be a low-cost, low-risk strategy, applicable to the primary care setting. Good instruction and demonstration ensured children mastered the technique and engaged with the treatment, but uncertainties were raised about training provision and potential impact on the GP consultation. Suitable educational materials and demonstrations of the autoinflation technique are likely to promote uptake and compliance. Making nasal balloon autoinflation part of a child's daily routine was seen to enhance compliance, but difficulties can arise if children are unwell or refuse to cooperate. Wider use of the nasal balloon has the potential to enhance early management of OME in primary care, and may help to fill the management gap arising from forthcoming changes to care pathways.

\section{Strengths and limitations}

This research is the first to provide pragmatic experiential data about use of the nasal balloon in primary care from the perspectives of both healthcare providers and parents who were early adopters of the method, and from whom important lessons of change can be learnt. GPs and nurses with hands-on experiences with the nasal balloon highlighted barriers and facilitators to prescribing and demonstrating the nasal balloon, and identified important strategies for implementation. Parents provided valuable insight into day-to-day usage of the nasal balloon in the home setting, which has not been previously captured in research studies. 
In routine clinical practice, GPs are likely to obtain information about the nasal balloon through published literature and online resources. Including GPs who had no previous experience or knowledge of the nasal balloon method facilitated exploration of views and potential concerns likely to arise in wider NHS practice. This qualitative study was conducted before the publication of the AIRS study, and the authors recognise that participant views may have differed had they known the outcome of the study.

This study included nurse recruiters who had dedicated research time to spend with families for OME management and instruction for use of the nasal balloon. The authors acknowledge that this may not reflect the available time and resource in routine primary care.

The parent sample represented more educated parents from areas of low social deprivation who were sufficiently interested in taking part in trials and may not have the same views as the general population. Finally, it would have been interesting to gather the views and experiences from the children themselves. Including children in research can enhance the scope and findings of a study. However, in this instance, the children were individually considered too young at aged $4-6$ years to be able to directly contribute to this qualitative work

\section{Comparison with existing literature}

Management options for children with OME are severely limited in primary care. According to meta-analyses, medical treatments such as antibiotics, antihistamines, decongestants, and intranasal corticosteroids are all ineffective, with significant associated harms, ${ }^{2-4}$ and therefore not recommended. The NICE guidelines currently recommend a 3-month monitoring period before consideration for grommet surgery. ${ }^{14} \mathrm{NHS}$ England has set out to reduce or stop the commissioning treatments where the risks outweigh any potential benefit, with a view to improving efficiencies and patient outcomes. Grommet surgery is one such treatment, and thus the care pathways for children with glue ear are likely to change. Better management strategies for clinically affected children in primary care are much needed, and nasal balloon autoinflation is really the only effective treatment that is applicable to a primary care setting..$^{10}$ Acceptability of the nasal balloon has previously been reported in three small secondary care studies, ${ }^{15-17}$ where the technique was described as 'fun ' or 'amusing' for the children. This is consistent with the findings of the authors study, where parents described the nasal balloon as a natural, 'holistic' treatment, which was 'acceptable' as a treatment for glue ear, and a 'novelty for the children: However, all parents had received detailed information about the treatment from a credible source (their GP or practice nurse) and were taking part in an ethically approved randomised controlled trial, and this could have affected their perception of the nasal balloon as a treatment.

There have been ongoing uncertainties about the age at which children can manage the nasal balloon treatment. GPs in this study, particularly those with no previous experience of the method, considered the nasal balloon treatment to be most suitable for older children. However, children recruited to AIRS were aged $\geq 4$ years, and overall compliance was reported as good, with $<3 \%$ of children unable to manage the technique. ${ }^{18}$ Previous studies have also reported that children aged $\geq 4$ years are generally able to master the technique, ${ }^{16,18}$ whereas children as young as 3 years can manage the technique with some instruction and encouragement. ${ }^{19}$ However, it is exceedingly unlikely that children aged 1-2 years would be able to use the nasal balloon, and for these children OME still represents a considerable challenge. Other middle ear insufflation devices have been suggested for the youngest age group, including the Politzer device, ${ }^{20}$ which requires less user-involvement than that for the nasal balloon but is more costly. Additionally, the Moniri ${ }^{\circledR}$ Otovent ${ }^{\circledR}$ (ABIGO Medical, Askim, Sweden) device ${ }^{21}$ may be used as either a Politzer or Valsalva device, though it may not be suitable for the very youngest affected children.

Healthcare professionals and parents in this study identified that the key to mastering the nasal balloon method relies on good instruction and demonstration. However, GPs are concerned that this may not be possible within a routine consultation, and may not be the best use of their time. Parents and children participating in AIRS ${ }^{10,18}$ were taught the technique by the research nurse and supported by an instruction video to view at home. Instruction videos have been found to improve inhaler techniques in children with asthma when used to back up the healthcare consultation. ${ }^{22}$ An evidencebased video for demonstrating the nasal balloon has the potential to reinforce the information provided by the healthcare professional during the consultation process. Treatment can typically last 1-3 months, though research has shown an effect as early as 2 weeks. ${ }^{16}$ Questions often arise about how long children can 


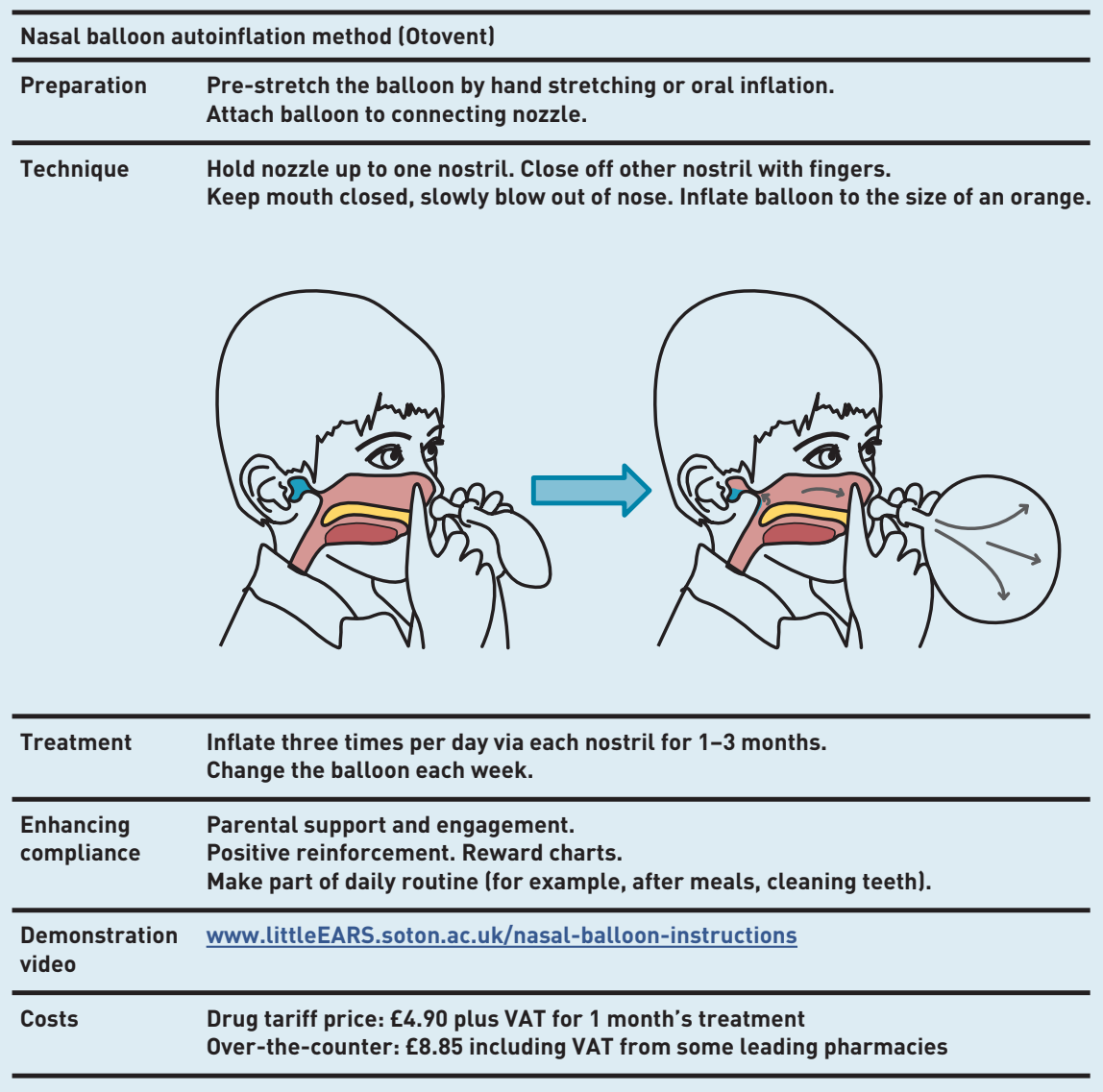

Figure 2. Nasal balloon autoinflation method.

persist with nasal balloon treatment. In the AIRS study, $89 \%$ of parents reported the use of the nasal balloon as 'most 'or 'all of the time' during the first month, and $80 \%$ up to 3 months. Although trial involvement might result in better compliance than routine practice, it does suggest that children are able to comply with treatment with parental support and engagement. In this qualitative work, parents reported that the key to remembering to use the nasal balloon was to make it part of the child's everyday routine, such as after cleaning their teeth or using their asthma inhaler. Routines and rituals are important organisers of family life. ${ }^{23}$ Children naturally adopt such routines as eating meals, daily homework, and bedtime. It has been theorised that adopting good routines can improve the likelihood of compliance to certain medical treatments. ${ }^{24}$ Adopting nasal balloon autoinflation as part of a routine may be very important for the longer-term use of the nasal balloon.

\section{Implications for research and practice}

This study suggests nasal balloon autoinflation is an acceptable treatment for early school-aged children with glue ear in primary care, and is feasible during a recommended 3-month monitoring period. In light of potential changes to care pathways for children with OME, the nasal balloon method offers a low-cost, low-risk management strategy for clinically affected children in primary care.

A brief demonstration of the nasal balloon method together with positive reinforcement by a health professional is likely to enhance child cooperation and improve overall adherence to the treatment schedule. The team has developed and evaluated an educational intervention and demonstration video that may help support families with use of the nasal balloon and thus minimise the work required of the healthcare provider (https://www.littleears. soton.ac.uk/nasal-balloon-instructions). ${ }^{25}$ The Handbook of Non-Drug Interventions (HANDI), ${ }^{26}$ developed by the Royal Australian College of General Practitioners, provides GPs with evidence-based information to promote non-medical interventions. Similar resources would be useful for UK general practice to support the wider prescription and uptake of treatments such as the nasal balloon method. The Otovent nasal balloon is currently available on prescription, or can be purchased online or at some leading pharmacists. Details of the method are presented in Figure 2.

Further research should explore the potential barriers to nasal balloon autoinflation in lower socioeconomic areas where OME may have greater developmental impact. 


\section{REFERENCES}

1. Zielhuis GA, Rach GH, Van den Broek P. The occurrence of otitis media with effusion in Dutch pre-school children. Clin Otolaryngol Allied Sci 1990; 15(2): 147-153.

2. Griffin G, Flynn CA. Antihistamines and/or decongestants for otitis media with effusion (OME) in children. Cochrane Database Syst Rev 2011; (9): CD003423. DOI: 10.1002/14651858.CD003423.pub3.

3. Simpson SA, Lewis R, van der Voort J, et al. Oral or topical nasal steroids for hearing loss associated with otitis media with effusion in children. Cochrane Database Syst Rev2011; (5): CD001935. DOI: 10.1002/14651858.CD001935.pub3.

4. Venekamp RP, Burton MJ, van Dongen TM, et al. Antibiotics for otitis media with effusion in children. Cochrane Database Syst Rev 2016; (6): CD009163. DOI: 10.1002/14651858.CD009163.pub3.

5. Burke P. Otitis media with effusion: is medical management an option? J R Coll Gen Pract 1989; 39(326): 377-382

6. NHS England, NHS Clinical Commissioners, Academy of Medical Royal Colleges, NHS Improvement, National Institute for Health and Care Excellence. Evidence-based interventions: consultation document. London: NHSE, NHSCC, AMRC, NHSI, NICE, 2018

7. Bennett KE, Haggard MP. Behaviour and cognitive outcomes from middle ear disease. Arch Dis Child 1999; 80(1): 28-35.

8. Silva PA, Chalmers D, Stewart I. Some audiological, psychological, educational and behavioral characteristics of children with bilateral otitis media with effusion: a longitudinal study. J Learn Disabil 1986; 19(3): 165-169.

9. Perera R, Glasziou PP, Heneghan CJ, et al. Autoinflation for hearing loss associated with otitis media with effusion. Cochrane Database Syst Rev 2013; (5): CD006285. DOI: 10.1002/14651858.CD006285.pub2.

10. Williamson I, Vennik J, Harnden A, et al. Effect of nasal balloon autoinflation in children with otitis media with effusion in primary care: an open randomized controlled trial. CMAJ 2015; 187(13): 961-969.

11. NHS Business Services Authority. Prescription cost analysis (PCA) data. 2018 https://uww.nhsbsa.nhs.uk/prescription-data/dispensing-data/prescriptioncost-analysis-pca-data laccessed 16 Nov 2018).

12. NHS Digital. Hospital admitted patient care activity, 2016-17. Leeds: NHSD, 2017

13. Braun V, Clarke V. Using thematic analysis in psychology. Qual Res Psychol
2006; 3(2): 77-101.

14. National Institute for Health and Care Excellence. Surgical management of otitis media with effusion in children. CG60. London: National Collaborating Centre for Women's and Children's Health, NICE, 2008.

15. Brooker DS, McNeice A. Autoinflation in the treatment of glue ear in children. Clin Otolaryngol Allied Sci 1992; 17(4): 289-290.

16. Stangerup SE, Sederberg-Olsen J, Balle V. Autoinflation as a treatment of secretory otitis media. A randomized controlled study. Arch Otolaryngol Head Neck Surg 1992; 118(2): 149-152.

17. Ercan I, Cakir B, Kayaoglu S, et al. Long term effect of autoinflation in the treatment of otitis media with effusion. KBB Forum 2005; 4(4): 166-170.

18. Williamson I, Vennik J, Harnden A, et al. An open randomised study of autoinflation in 4- to 11-year-old school children with otitis media with effusion in primary care. Health Technol Assess 2015; 19(72): 1-150.

19. Stangerup SE, Tjernstrom 0 , Harcourt J, et al. Barotitis in children after aviation; prevalence and treatment with Otovent. J Laryngol Otol 1996; 110(7): 625-628.

20. Banigo A, Hunt A, Rourke T, et al. Does the EarPopper ${ }^{\circledR}$ device improve hearing outcomes in children with persistent otitis media with effusion? A randomised single-blinded controlled trial. Clin Otolaryngol 2016; 41(1): 59-65.

21. Bidarian-Moniri A, Ramos MJ, Goncalves I, Ejnell H. A new device for treatment of persistent otitis media with effusion. Int J Pediatr Otorhinolaryngol 2013; 77(12): 2063-2070

22. Carpenter DM, Lee $\mathrm{C}, \mathrm{Blalock} \mathrm{SJ}$, et al. Using videos to teach children inhaler technique: a pilot randomized controlled trial. J Asthma 2015; 52(1): 81-87.

23. Bossard J, Boll E. Ritual in family living. A contemporary study. Philadelphia, PA: University of Pennsylvania Press, 1950.

24. Fiese BH, Wamboldt FS, Anbar RD. Family asthma management routines: connections to medical adherence and quality of life. J Pediatr 2005; 146(2): 171-176.

25. Vennik J. Development of an educational intervention to support implementation of nasal balloon autoinflation for glue ear: a mixed methods approach. [Doctoral thesis]. 2017. https://eprints.soton.ac.uk/415999/ laccessed 16 Nov 2018).

26. Royal Australian College of General Practitioners. The HANDI project. 2018 https://www.racgp.org.au/your-practice/guidelines/handi/about/the-handiproject/ (accessed 16 Nov 2018). 